\title{
Suppression of Exciton-Electron Scattering in Doped Single-Walled Carbon Nanotubes
}

\author{
S. Konabe, ${ }^{1,3}$ K. Matsuda, ${ }^{2}$ and S. Okada ${ }^{1,3}$ \\ ${ }^{1}$ Graduate School of Pure and Applied Sciences, University of Tsukuba, Tsukuba, Ibaraki 305-8571, Japan \\ ${ }^{2}$ Institute of Advanced Energy, Kyoto University, Gokasho, Uji, Kyoto 611-0011, Japan \\ ${ }^{3}$ Japan Science and Technology Agency, CREST, 7 Gobancho, Chiyoda, Tokyo 102-0076, Japan
}

(Received 25 January 2012; published 31 October 2012)

\begin{abstract}
We theoretically study the phase relaxation of excitons in doped single-walled carbon nanotubes caused by elastic scattering between an exciton and an electron. Our calculation of the spectral linewidth, that is the inverse of the scattering rate, demonstrates that this dephasing process is unexpectedly suppressed in doped single-walled carbon nanotubes, giving rise to anomalous nonlinear behavior as a function of the electron density. A detailed investigation reveals that the logarithmic correction of the exciton self-energy plays a critical role in the exciton-carrier scattering dynamics.
\end{abstract}

PACS numbers: 78.67.Ch, 71.35.Cc, 78.20.Bh

Photoexcited states of single-walled carbon nanotubes (SWCNTs) are characterized by a quasiparticle comprising an electron and a hole, i.e., an exciton, due to the strong Coulomb interaction caused by the one-dimensional tubular structure [1-3]. The binding energy of an exciton is a few hundreds of meV. Due to this large binding energy, the exciton is known to play a crucial role in determining the optical response of the SWCNTs [4]. Strong Coulomb interaction further leads to another bound state called a trion that consists of an exciton and a charged carrier [5,6]. These novel quasiparticles open a new paradigm of manybody physics in nanoscale materials, leading to unexpected physical phenomena that are remarkably different from conventional many-particle systems in bulk materials. The novel physical phenomena caused by interacting excitons in SWCNTs give deeper insight into many-body physics and extend the range of optical or optoelectronic technologies [7-9].

The emergence of a novel bound state raises the question as how strong Coulomb interaction in low-dimensional materials affects the dynamical properties of many-body exciton states and the exciton-carrier state. Recently, it was experimentally demonstrated that such many-body effects profoundly influence the dynamics of excitons in SWCNTs, showing a highly nonlinear optical response over a wide range of excitation power intensities [10-13]. It was found that phase relaxation by elastic scattering among excitons is the dominant contribution to the linewidths of optical spectra [14], larger than the contribution of Auger exciton recombination caused by inelastic scattering among the excitons [15-17]. For doped SWCNTs, besides elastic scattering between excitons, similar to compound semiconductors [18], it is also expected that elastic scattering between excitons and charged carriers plays a crucial role for determining the phase relaxation of excitons. On the other hand, Perebeinos and Avouris discussed the carrier density dependence of exciton-phonon scattering in doped SWCNTs [19]. This scattering leads to the population relaxation of excitons, although the scattering rates by this process are negligibly small.

In this study, we carry out a theoretical investigation of the dephasing process caused by elastic scattering between excitons and electrons to unravel the dynamical processes of excited states in doped SWCNTs. Our calculation predicts that this scattering process shows, contrary to expectation, strong suppression and nonlinearity associated with the doped electron density. We reveals that this anomalous behavior is caused by a cusp-like structure in the dispersion relation of excitons that originates from the logarithmic correction in the exciton self-energy, which itself can be traced to the unscreened exchange interaction between electrons and holes. Our results show that the exciton dynamics is strongly influenced by the unscreened exchange interaction.

To start, we calculate the collisional broadening of the spectral linewidth associated with the elastic excitonelectron scattering process. In particular, we focus on the scattering process of an electron and a spin-singlet exciton of the lowest energy, which is the only optically allowed exciton (i.e., a bright exciton) in SWCNTs and usually denoted by $E_{11}$. The linewidth, determined as the dephasing rate, can be calculated by Fermi's golden rule,

$$
\begin{aligned}
\Gamma= & 2 \pi \sum_{k, q}|M(k, q)|^{2} \delta\left(\left(E(0)+\varepsilon_{c}(k)-E(q)\right.\right. \\
& \left.-\varepsilon_{c}(k-q)\right) f(k)[1-f(k-q)],
\end{aligned}
$$

where $E(k)$ is the band energy of the exciton obtained from solving the Bethe-Salpeter equation and $\varepsilon(k)$ stands for the electron energy. The terms $f(k)$ and $1-f(k-q)$ are the Fermi distribution functions for incoming scattering electrons and for outgoing scattered electrons, respectively, while Fig. 1 diagrammatically shows the matrix element $M(k, q)$ of the exciton-electron scattering process [20].

The exciton amplitude $Z_{k, q}^{n}$ and energy $E^{n}(q)$ are obtained by solving the Bethe-Salpeter equation $[1,2,21,22]$ : 


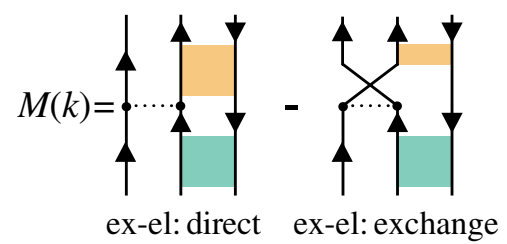

FIG. 1 (color online). The matrix element of the excitonelectron scattering. The arrows indicate electrons or holes. The dotted lines stand for the Coulomb interaction, and the squares mark exciton states.

$\left[\varepsilon_{c}(k+q)-\varepsilon_{v}(k)\right] Z_{k, q}^{n}+\sum_{k^{\prime}} K_{k, k^{\prime}} Z_{k^{\prime}, q}^{n}=E^{n}(q) Z_{k, q}^{n}$

where $K_{k, k^{\prime}} \equiv K_{k, k^{\prime}}^{\mathrm{ex}}-K_{k, k^{\prime}}^{\mathrm{dir}}$ is the Coulomb interaction kernel that consists of exchange $\left(K_{k, k^{\prime}}^{\mathrm{ex}}\right)$ and screened-direct $\left(K_{k, k^{\prime}}^{\mathrm{dir}}\right)$ terms. The exchange interaction contributes only to the singlet exciton. For the Coulomb potential between $\pi$ orbitals, we employ the Ohno potential $V(\mathbf{r})=U / \kappa \sqrt{\left(\frac{4 \pi \epsilon_{0}}{e^{2}} U|\mathbf{r}|\right)^{2}+1} \quad($ with $\quad U=11.3 \mathrm{eV}$ ) $[2,21,22]$. The quasiparticle energies $\varepsilon_{c}(k)$ and $\varepsilon_{v}(k)(c$ and $v$ indicate conduction and valence bands, respectively) are calculated by applying the random-phase approximation to the dielectric function of $\pi$ electrons [1,21,22]. We further choose a dielectric constant of $\kappa=3.3$ to incorporate the screening effects by core states, $\sigma$ electrons and the surrounding environment [19], and the calculations are performed using the tight-binding approximation with a nearest-neighbor hopping of $3.0 \mathrm{eV}$ [2]. In Eq. (1), $E(k) \equiv E^{1}(k)$.

Figure 2 shows the linewidth of the optical spectra of the singlet bright exciton for semiconducting zigzag SWCNTs ranging from $(13,0)$ to $(23,0)$ at temperatures of $50 \mathrm{~K}$ and $300 \mathrm{~K}$ as a function of the electron density. The solid and dashed lines are type I $[\bmod (2 n+m, 3)=1]$ and type II $[\bmod (2 n+m, 3)=2]$ for $(n, m)$ SWCNTs, respectively. We find two interesting features at the lower temperature of $50 \mathrm{~K}$ : (i) the linewidth is strongly suppressed compared with the one at $300 \mathrm{~K}$, which indicates that an exciton is less scattered by a doped electron at the lower temperature, and (ii) the linewidth increases nonlinearly with increasing electron density before peaking and saturating (see the inset of Fig. 2). This behavior in SWCNTs is quite unusual compared with that of other conventional semiconductors. It is well known that the linewidth of conventional semiconductors in both bulk and nanoscale materials becomes larger at lower temperatures [20] and is proportional to the electron density for any temperature at low density $[18,23,24]$. In contrast to the low temperature behavior at $50 \mathrm{~K}$, the linewidth of the exciton at $300 \mathrm{~K}$ exhibits a linear dependence on electron density in the low density region. Although we focus here only on zigzag SWCNTs for simplicity, the results do not depend on the chirality of semiconducting SWCNTs.

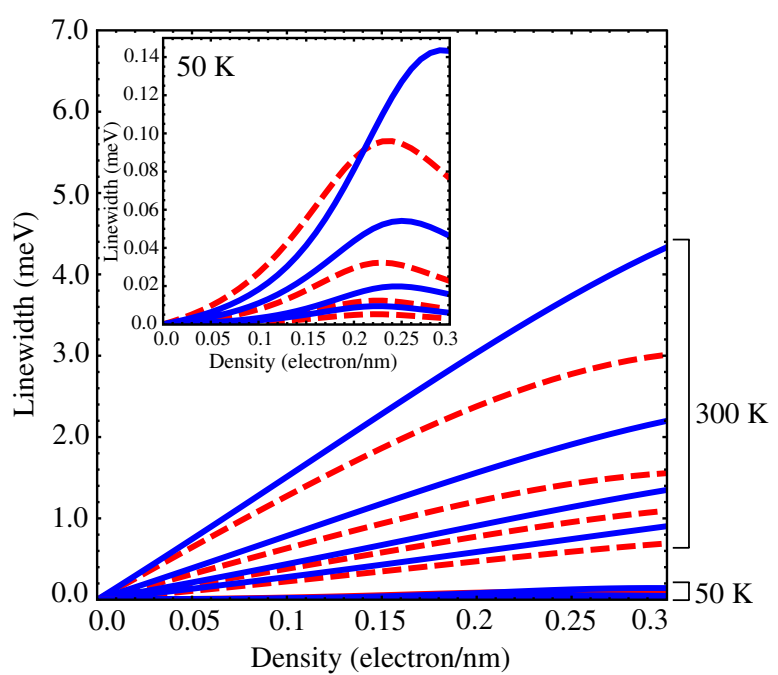

FIG. 2 (color online). Electron density dependence of the collisional linewidth of SWCNTs at 300 and $50 \mathrm{~K}$. The inset shows the expansion of the linewidth at $50 \mathrm{~K}$. In each panel, the solid and dashed lines are Type I $[(14,0),(17,0),(20,0)$, and $(23,0)$ SWCNTs from the top to the bottom $]$ and Type II $[(13,0)$, $(16,0),(19,0)$, and $(22,0)$ SWCNTs from the top to the bottom], respectively.

We ascribe the unusual behavior of the linewidth to the anomalous energy spectrum of the singlet bright exciton: As shown in Fig. 3(a), the energy dispersion of the singlet exciton is no longer parabolic but has a $k^{2} \log (k)$ correction leading to a cusplike structure $[22,25,26]$. This anomalous band structure originates from the unscreened electronhole exchange interaction $K_{k, k^{\prime}}^{\mathrm{ex}}$ in the Coulomb kernel of Eq. (2), which induces a strong renormalization of the exciton effective mass [25,27]. Similarly, the unscreened exchange interaction at the Dirac point in undoped graphene gives a strongly renormalized dispersion relation which is a signature of a marginal Fermi liquid [25,27-29]. Under this unusual dispersion, the energy-momentum conservation for electrons and excitons $\left(E(0)+\varepsilon_{c}(k)-\right.$ $\left.E(q)-\varepsilon_{c}(k-q)=0\right)$ is difficult to satisfy due to the lack of available electrons states below the cusplike structure, giving rise to the anomalous scattering between excitons and electrons.

Let us discuss in more detail the underlying kinematics. The Fermi distribution determines the energy region where the incoming electrons in Eq. (1) with energy $\varepsilon_{c}(k)$ are available, as shown in Figs. 3(b) and 3(c). As in Fig. 3(b), it is demonstrated that within such energy region of the incoming electron, the outgoing electron with energy $\varepsilon_{c}(k-q)$ where the dispersion is below the cusplike structure of the exciton dispersion does not have a possible final state. This arises totally from the anomalous band structure of the singlet exciton. This effect on the phase-space kinematics leads to a restriction on the scattering processes, resulting in a decrease in the number of states in the summation in Eq. (1). Therefore, nonlinearity of the 
(a)

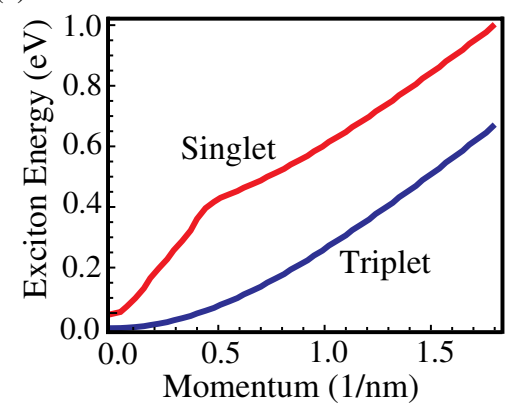

(b)

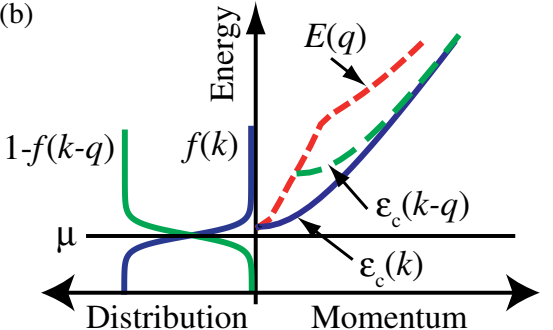

(c)

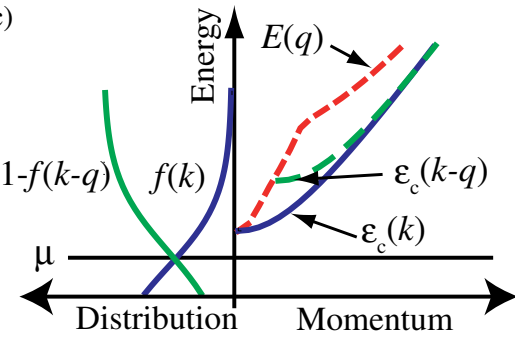

FIG. 3 (color online). (a) The band energies of singlet bright exciton (red line) and triplet dark exciton (blue line) of $(13,0)$ SWCNTs. The energy is measured from the band edge of the triplet dark exciton. (b) and (c) The energy-momentum relation of exciton-electron scattering processes at lower temperatures (b) and at higher temperatures (c) The figures on the left are schematic distribution functions for incoming $(f)$ and outgoing $(1-f)$ electrons with chemical potential $\mu$. The figures on the right are the energy dispersions of incoming electrons (blue solid line), outgoing electrons (green long-dashed line), and singlet bright excitons (red dashed line).

linewidth associated with suppression of scattering emerges. This is clearly observed at low temperatures, as shown in Fig. 3(b). In contrast, with increasing temperature, the available states for outgoing electrons in the summation in Eq. (1) increase, so that the scattering rate becomes finite even at a low electron density, as schematically shown in Fig. 3(c). Indeed, at higher temperatures, the scattering rate recovers the usual linear behavior with an increase in the electron density, as shown in Fig. 2.

When the incoming electron energy is in the region where the exciton band possesses nearly parabolic dispersion, the effect of the exchange self-energy is less important. As a result, final states for outgoing electrons are sufficiently available, leading to finite scattering probability. Further increasing the electron density leads to sublinear behavior of the linewidth. In this regime, the final scattered states are already filled by doped electrons.
Therefore the electron is prohibited from the scattering as a result of the Pauli exclusion principle (phase-space filling effects) [20].

It is important for complete treatments of doping effects to consider many-body effects by including dynamical screening effects mediated by acoustic plasmons [30] and the effect of strongly bound charged excitons [5,31]. Those are peculiar features in doped semiconducting carbon nanotubes. In the present study, however, we discussed the exciton-electron scattering based on the non-correlated excitons that is obtained from Eq. (2). This approximation works well in the low-density region where we can safely ignore the screening by doped electrons. This density region is much below the degenerate limit of doped electrons. Within this condition, we do not expect our results to be dramatically influenced by dynamical screening effects.

So far, we have discussed the elastic scattering between an electron and a singlet bright exciton. For comparison, we discuss the elastic scattering between an electron and an optically inactive exciton (i.e., a dark exciton), especially a spin-triplet exciton, that has a normal parabolic band structure [Fig. 3(a)]. Figure 4 shows the linewidth of triplet dark excitons in $(13,0)$ SWCNTs at 50 and $300 \mathrm{~K}$. In contrast to the scattering processes of the singlet exciton, the scattering in this case occurs linearly up to the degenerate density of electrons leading to the common linear density dependence of the linewidth [23,24,32]. This is simply because there always exists a scattering process that satisfies the energy-momentum conservation. The temperature dependence of the linewidth of the triplet exciton before saturation is opposite to that of the bright exciton; the linewidth is larger at lower temperatures. This agrees with the case of semiconductor quantum wells where the linewidth becomes larger at low temperatures [20]. From the above difference between singlet and triplet

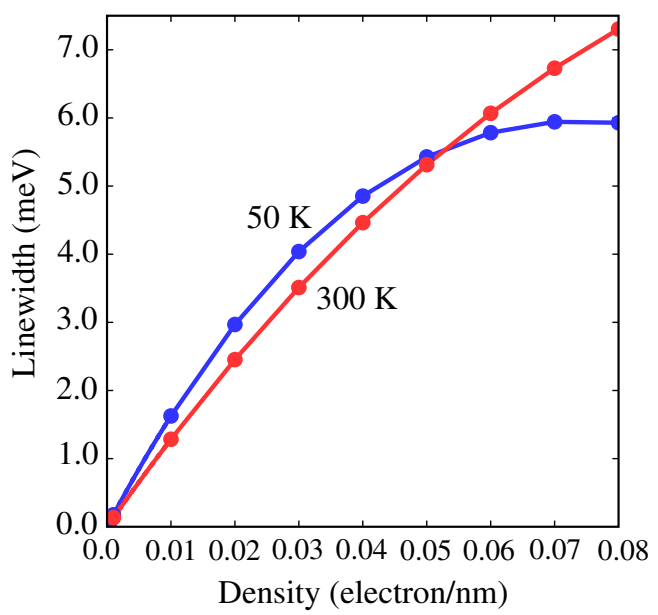

FIG. 4 (color online). Collisional linewidth of the elastic scattering between the triplet dark exciton and electrons for $(13,0)$ SWCNTs at a temperature of $50 \mathrm{~K}$ (blue line) and $300 \mathrm{~K}$ (red line). 


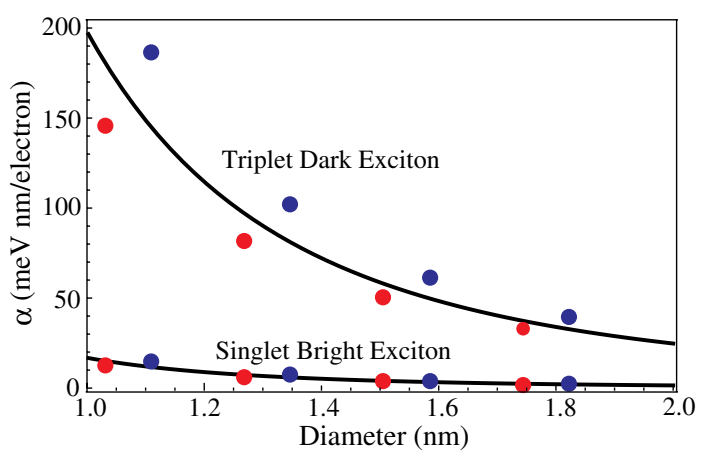

FIG. 5 (color online). The diameter dependence of $\alpha(d)$ for the singlet bright and triplet dark excitons, where upper blue (lower red) points indicate $\alpha(d)$ for type I (II) semiconducting zigzag SWCNTs. The solid lines are fitted by $d^{-3.5}\left(d^{-3}\right)$ for the scattering of singlet bright (triplet dark) excitons.

excitons, it is apparent that the exchange interaction of the kernel in the Bethe-Salpeter equation (2) is crucial for exciton-electron scattering.

Finally, we discuss the linewidth in the linear region for both the singlet and triplet excitons at $300 \mathrm{~K}$. In this region, the scattering rate is expressed by the linear function of electron density as

$$
\Gamma(d)=\alpha(d) n,
$$

where $\alpha(d)$ is the coefficient as a function of the diameter of the SWCNTs and $n$ is the electron density. Figure 5 shows the calculation results of $\alpha(d)$ for the singlet bright and triplet dark excitons, where upper (lower) points indicate $\alpha(d)$ for type I (II) semiconducting zigzag SWCNTs for each plot. We can fit $\alpha(d)$ in Eq. (3) as $\alpha(d) \propto d^{-3.5}$ for the bright (singlet) exciton at $300 \mathrm{~K}$, and as $\alpha(d) \propto d^{-3}$ for the dark (triplet) excitons at $300 \mathrm{~K}$ as shown in Fig. 5. The fitting formula for the triplet dark exciton $\left[\alpha(d) \propto d^{-3}\right]$ agrees with the analytical result of the effective mass approximation with an attractive contact potential [33,34], which gives $\alpha(d) \propto E_{\mathrm{b}}^{2} M_{\mathrm{ex}} \propto d^{-3}$, where $E_{b}\left(\propto d^{-1}\right)$ and $M_{\mathrm{ex}}\left(\propto d^{-1}\right)$ are the binding energy and the effective mass of the exciton, respectively. For the singlet bright exciton, the exchange interaction makes it difficult to obtain an analytical result. However, we expect that the different exponent of the diameter dependence originates with the anomalous energy dispersion.

The coefficient $\alpha(d)$ can be also written as $\gamma a_{\mathrm{B}} E_{b}$ [24], where $\gamma$ is a dimensionless parameter that represents the strength of exciton-electron scattering and $a_{\mathrm{B}}$ is the exciton Bohr radius. From this relation, we obtain $\gamma=0.05$ for the singlet bright exciton of, for instance, $E_{b}=400 \mathrm{meV}$ and $a_{\mathrm{B}}=1 \mathrm{~nm}$ at $300 \mathrm{~K}$. From the absorption spectral changes in hole doped SWCNTs, it has experimentally been estimated recently that $\gamma \simeq 0.08$ [35], which is in good agreement with our theoretical result. For the triplet dark exciton at $300 \mathrm{~K}$, we obtain $\gamma=0.5$ that is ten times larger than that of the bright exciton scattering. From the above difference of the diameter dependence and the scattering strength between the bright and dark exciton, we infer that the different final density of states of scattering processes is crucial even in the linear region of the singlet linewidth at higher temperature.

It has been argued that the unscreened exchange interaction determines the static properties of excited states of SWCNTs such as the fine structure of exciton states [21] or the the extremely large energy separation between the exciton and trion [5]. In addition to such static properties, our calculation reveals that the dynamical properties of excitons in SWCNTs are also profoundly influenced by the unscreened exchange interaction via the logarithmic correction to the exciton dispersion.

In summary, we have investigated the collisional broadening of the linewidth by exciton-electron scattering in doped SWCNTs. Our calculation shows that the density dependence of the linewidth exhibits anomalous nonlinear behavior. This indicates that the exciton-electron scattering process in SWCNTs is very peculiar compared to that in other semiconductors. We interpreted this behavior based on the anomalous structure of the exciton band, which originates in the unscreened exchange self-energy between electrons and holes. Because of the characteristic exciton dispersion, exciton-electron scattering is forbidden, leading to suppression of the exciton-electron scattering. Our calculation also gives an insight into the formation mechanism of trions in SWNCTs. Since our conclusion is that the exciton and free carrier are less scattered, it seems to be difficult to form trions from exciton and free carriers, for instance, by the electrostatic doping [36]. This may indicate that localized states of carriers are necessary and crucial for trion formation.

We would like to thank S. Mouri for discussion and C. M. Puetter for critical reading of our manuscript. This work was supported by CREST of the Japan Science and Technology Agency and a Grant-in-Aid for Scientific Research from the Ministry of Education, Culture, Sports, Science and Technology (MEXT) of Japan, and by the "Joint Usage/Research Program on Zero-Emission Energy Research, Institute of Advanced Energy, Kyoto University (B-19)."

[1] T. Ando, J. Phys. Soc. Jpn. 66, 1066 (1997).

[2] V. Perebeinos, J. Tersoff, and P. Avouris, Phys. Rev. Lett. 92, 257402 (2004).

[3] C.D. Spataru, S. Ismail-Beigi, L. X. Benedict, and S. G. Louie, Phys. Rev. Lett. 92, 077402 (2004).

[4] F. Wang, G. Dukovic, L. E. Brus, and T. F. Heinz, Science 308, 838 (2005).

[5] R. Matsunaga, K. Matsuda, and Y. Kanemitsu, Phys. Rev. Lett. 106, 037404 (2011).

[6] S. M. Santos, B. Yuma, S. Berciaud, J. Shaver, M. Gallart, P. Gilliot, L. Cognet, and B. Lounis, Phys. Rev. Lett. 107, 187401 (2011). 
[7] P. Avouris, Z. Chen, and V. Perebeinos, Nature Nanotech. 2, 605 (2007).

[8] P. Avouris, M. Freitag, and V. Perebeinos, Nature Photon. 2, 341 (2008).

[9] C. Galland and A. Imamoğlu, Phys. Rev. Lett. 101, 157404 (2008).

[10] K. Matsuda, T. Inoue, Y. Murakami, S. Maruyama, and Y. Kanemitsu, Phys. Rev. B 77, 033406 (2008).

[11] Y. Murakami and J. Kono, Phys. Rev. Lett. 102, 037401 (2009).

[12] Y.F. Xiao, T. Q. Nhan, M. W. B. Wilson, and J. M. Fraser, Phys. Rev. Lett. 104, 017401 (2010).

[13] S. Moritsubo, T. Murai, T. Shimada, Y. Murakami, S. Chiashi, S. Maruyama, and Y. K. Kato, Phys. Rev. Lett. 104, 247402 (2010).

[14] D. T. Nguyen, C. Voisin, Ph. Roussignol, C. Roquelet, J. S. Lauret, and G. Cassabois, Phys. Rev. Lett. 107, 127401 (2011).

[15] F. Wang, G. Dukovic, E. Knoesel, L.E. Brus, and T.F. Heinz, Phys. Rev. B 70, 241403(R) (2004).

[16] Y.-Z. Ma, L. Valkunas, S. L. Dexheimer, S. M. Bachilo, and G. R. Fleming, Phys. Rev. Lett. 94, 157402 (2005).

[17] L. Huang and T. D. Krauss, Phys. Rev. Lett. 96, 057407 (2006).

[18] L. Schultheis, J. Kuhl, A. Honold, and C. W. Tu, Phys. Rev. Lett. 57, 1635 (1986).

[19] V. Perebeinos and P. Avouris, Phys. Rev. Lett. 101, 057401 (2008).

[20] G. Ramon, A. Mann, and E. Cohen, Phys. Rev. B 67, 045323 (2003).

[21] T. Ando, J. Phys. Soc. Jpn. 75, 024707 (2006).
[22] J. Jiang, R. Saito, Ge. G. Samsonidze, A. Jorio, S. G. Chou, G. Dresselhaus, and M. S. Dresselhaus, Phys. Rev. B 75, 035407 (2007).

[23] A. Honold, L. Schultheis, J. Kuhl, and C. W. Tu, Phys. Rev. B 40, 6442 (1989).

[24] R. Kumar, A. S. Vengurlekar, A. V. Gopal, T. Mélin, F. Laruelle, B. Etienne, and J. Shah, Phys. Rev. Lett. 81, 2578 (1998).

[25] V. Perebeinos, J. Tersoff, and P. Avouris, Nano Lett. 5, 2495 (2005).

[26] M. S. Dresselhaus, G. Dresselhaus, R. Saito, and A. Jorio, Annu. Rev. Phys. Chem. 58, 719 (2007).

[27] R. M. Konik, Phys. Rev. Lett. 106, 136805 (2011).

[28] J. González, F. Guinea, and M. A. H. Vozmediano, Phys. Rev. B 59, R2474 (1999).

[29] C. L. Kane and E. J. Mele, Phys. Rev. Lett. 93, 197402 (2004).

[30] C. D. Spataru and F. Léonard, Phys. Rev. Lett. 104, 177402 (2010).

[31] K. Watanabe and K. Asano, Phys. Rev. B 85, 035416 (2012).

[32] Y.P. Feng and H. N. Spector, Superlattices Microstruct. 3, 459 (1987).

[33] F. Wang, Y. Wu, M. S. Hybertsen, and T. F. Heinz, Phys. Rev. B 73, 245424 (2006).

[34] J. M. Kinder and E. J. Mele, Phys. Rev. B 78, 155429 (2008).

[35] S. Mouri and K. Matsuda, J. Appl. Phys. 111, 094309 (2012).

[36] S. Yasukochi, T. Murai, S. Moritsubo, T. Shimada, S. Chiashi, S. Maruyama, and Y. K. Kato, Phys. Rev. B 84, 121409 (2011). 